\section{OPEN ACCESS}

Edited by:

loannis P. Androulakis, Rutgers, The State University of New Jersey, USA

Reviewed by:

Litao Sun,

The Scripps Research Institute, USA

Hisham Bahmad,

American University of Beirut,

Lebanon

${ }^{*}$ Correspondence:

Jan Borén

jan.boren@wlab.gu.se

Specialty section: This article was submitted to

Systems Biology,

a section of the journal

Frontiers in Physiology

Received: 31 August 2015 Accepted: 03 November 2015 Published: 20 November 2015

Citation:

Adiels M, Mardinoglu A, Taskinen M-R and Borén J (2015) Kinetic Studies to

Elucidate Impaired Metabolism of Triglyceride-rich Lipoproteins in Humans. Front. Physiol. 6:342 doi: 10.3389/fphys.2015.00342

\title{
Kinetic Studies to Elucidate Impaired Metabolism of Triglyceride-rich Lipoproteins in Humans
}

\begin{abstract}
Martin Adiels ${ }^{1,2}$, Adil Mardinoglu ${ }^{3,4}$, Marja-Riitta Taskinen ${ }^{5}$ and Jan Borén ${ }^{1 *}$
${ }^{1}$ Department of Molecular and Clinical Medicine/Wallenberg Laboratory, University of Gothenburg, Gothenburg, Sweden, ${ }^{2}$ Health Metrics Unit, Sahlgrenska Academy, University of Gothenburg, Gothenburg, Sweden, ${ }^{3}$ Department of Biology and Biological Engineering, Chalmers University of Technology, Gothenburg, Sweden, ${ }^{4}$ Science for Life Laboratory, KTH - Royal Institute of Technology, Stockholm, Sweden, ' Heart and Lung Centre, Helsinki University Hospital and Research Programs' Unit, Diabetes \& Obesity, University of Helsinki, Helsinki, Finland
\end{abstract}

To develop novel strategies for prevention and treatment of dyslipidemia, it is essential to understand the pathophysiology of dyslipoproteinemia in humans. Lipoprotein metabolism is a complex system in which abnormal concentrations of various lipoprotein particles can result from alterations in their rates of production, conversion, and/or catabolism. Traditional methods that measure plasma lipoprotein concentrations only provide static estimates of lipoprotein metabolism and hence limited mechanistic information. By contrast, the use of tracers labeled with stable isotopes and mathematical modeling, provides us with a powerful tool for probing lipid and lipoprotein kinetics in vivo and furthering our understanding of the pathogenesis of dyslipoproteinemia.

Keywords: very low density lipoproteins, apoB, multocomnpartmental modeling, kinetics, stable isotopes

\section{INTRODUCTION}

The most abundant lipids in plasma are: triglycerides, cholesterol, cholesterol esters, and phospholipids. Since lipids are water-insoluble they have to be transported in lipoprotein particles. They consist of a hydrophobic core of triglycerides and cholesterol esters, shielded from the water by a surface monolayer of phospholipids, unesterified cholesterol, and specific proteins (Mahley et al., 1984). The protein components of the lipoprotein are known as apolipoproteins (apo) (Mahley et al., 1984). The amount of lipids and proteins in the lipoprotein particles affect their density - the lower the density of a lipoprotein, the more lipids it contains relative to protein. Depending on function and hydrated density, the lipoproteins are traditionally divided into four major classes. These are chylomicrons, very low-density lipoproteins (VLDL), low-density lipoproteins (LDL), and high-density lipoprotein (HDL).

Chylomicrons and VLDL particles are the major carriers of triglycerides in the circulation. Chylomicrons are synthesized in the intestine and carry dietary lipids absorbed by the intestine. VLDL particles are synthesized by the liver. The function of these lipoprotein particles is to transport and deliver triglycerides to adipose tissue and muscles. Elevated triglycerides in plasma are associated with increased risk for cardiovascular disease (CVD).

In order to prevent and treat disturbances in metabolism of triglyceride-rich lipoproteins, it is necessary to clarify the underlying mechanism(s). The hypertriglyceridemia can either be caused by increased secretion, conversion, or catabolism of lipoprotein particles of triglyceride-rich lipoproteins. Although static s measurements of plasma lipids and functional assays may give some information, in the end, it is necessary to study the true unit of function (the integrated metabolic 
pathway) to understand the complexity of lipoprotein metabolism (Chan et al., 2004a,b; Adiels et al., 2008). Therefore, kinetic studies with stable isotopes are critical to explore and clarify the pathophysiology of lipid disorders in humans (Chan et al., 2004a,b; Adiels et al., 2008). The aim of this review is to illustrate how kinetic studies have furthered our understanding of impaired human lipoprotein metabolism.

\section{METABOLISM OF apoB-CONTAINING LIPOPROTEINS}

Triglyceride-rich lipoproteins in the circulation are a mixture of chylomicrons (synthesized in the intestine) and VLDL particles (synthesized in the liver) (Figure 1). Each of these lipoproteins contains one molecule of apolipoprotein $\mathrm{B}$ (apoB). apoB is a large hydrophobic protein that remains bound to the lipoprotein particles (Segrest et al., 2001). This unique characteristic of apoB makes it possible to use apoB as a tool to trace the intravascular kinetics of the triglyceride-rich lipoproteins.

apoB is present in two different lengths; apoB100 and apoB48. The shorter form apoB48 corresponds to the amino-terminal $48 \%$ of apoB100. It is in humans exclusively synthesized in the intestine, and thus present on intestinal-derived chylomicrons and their remnants. The longer form, apoB100 is synthesized in the liver and present on VLDL, IDL, and LDL. Both apoB100 and apoB48 are coded by the same gene, but the shorter apoB48 is generated as a result of a posttranscriptional process called "RNA editing" that converts a cytidine-to-uridine (C-to-U) that generates a stop-codon and thus a truncated form of the fulllength protein. In humans, this posttranscriptional RNA editing occurs in the intestine only but in certain animals such as rodents and dogs, the process occurs also in the liver (Powell et al., 1987).

Why is apoB48 synthesized by this complex mechanism in the Intestine? The explanation is still unclear but it has been shown that apoB48-containing lipoproteins can carry more lipids than apoB100-containing lipoproteins. This is important since the chylomicrons must have capacity to rapidly and efficiently absorb large amounts of dietary lipids and a meal (Hussain, 2014). Once synthesized, chylomicrons are secreted into the lymphatic vessels until they enter into the bloodstream close to the heart. Thus, they are delivered directly to adipose tissue and muscles without first being metabolized by the liver. In the circulation, chylomicrontriglycerides are hydrolyzed by the enzyme lipoprotein lipase, which is present on the endothelial cells in the heart, muscle, and adipose tissue. The released free fatty acids are then taken up these tissues where they are stored or used for energy production. The regulation is LPL is controlled transcriptionally and posttranscriptionally. On the endothelial cells, LPL is activated by apoC-II and inhibited by apoC-III and ANGPTL4.

When the triglycerides are removed from the hydrophobic core of the chylomicrons, they shrink in size and become chylomicron remnants. The smaller chylomicron remnants are cleared from the circulation by the liver (Mahley and Ji, 1999; MacArthur et al., 2007; Williams, 2008; Williams and Chen, 2010). Recent studies have shown that chylomicron remnants are atherogenic and directly involved in atherogenesis. The explanation for this is that chylomicrons in addition to triglycerides also contain some cholesterol esters. When triglycerides are removed from the lipoprotein particle, the cholesterol esters remain. Thus, the chylomicron remnants become enriched in cholesteryl esters.

The liver secretes apoB100-containing VLDL particles. In fact, the liver produces two different forms of VLDL; larger VLDL and smaller $\mathrm{VLDL}_{2}$ particles. The triglyceride-rich $\mathrm{VLDL}_{1}$ particles carry most of the plasma triglycerides and have been shown to be the major determinant for the variation of plasma triglycerides in both healthy subjects and individuals with type 2 diabetes (Hiukka et al., 2005; Boren et al., 2014; Taskinen and Boren, 2015). The formation of $\mathrm{VLDL}_{1}$ is highly associated with fatty liver (Adiels et al., 2008; Boren et al., 2013). Indeed, subjects with non-alcoholic fatty live disease (NAFLD) have increased $\mathrm{VLDL}_{1}$ production (Adiels et al., 2006b).

VLDL-triglycerides are hydrolyzed by the same LPLdependent mechanisms as chylomicrons, Figure 1. As VLDL-triglycerides are hydrolyzed, the density of the lipoprotein increases and they become intermediate density lipoproteins (IDL) and subsequently LDL. Like chylomicrons, also VLDL contains mainly triglycerides but also some cholesterol esters that become enriched in the lipoprotein particle when the triglycerides are removed. Thus, the end-product of the lipolytic cascade, LDL contains mainly cholesterol esters and is the major determinant of cholesterol in plasma. LDL particles are removed from the circulation by the LDL-receptor on the hepatocytes. The importance of the LDL-receptor for clearance of LDL particles is illustrated by the genetic disorder, familial hypercholesterolemia caused by mutation in the LDL-receptor. The disease is associated with hypercholesterolemia and premature CVD.

Patients with obesity and insulin resistance have a characteristic atherogenic dyslipidemia characterized by increased plasma triglycerides, excessive postprandial lipemia (i.e., rise in triglyceride-rich lipoproteins after eating) postprandial hyperlipidemia, and low concentrations of HDL cholesterol (Taskinen, 2003; Adiels et al., 2006a). Interestingly, these lipid disturbances are not isolated abnormalities but metabolically linked to each other (Taskinen, 2005), and they appear years before type 2 diabetes is diagnosed (Taskinen, 2003).

\section{PRINCIPLES OF TRACER METHODOLOGY}

To simplest approach to study the kinetics of a molecule (i.e., the tracee) is to introduce the same molecule (tracer), but labeled into the system (Chan et al., 2004a,b). This process has many advantages but also obvious concerns, especially in human studies. The alternative is to introduce a labeled precursor of the molecule of interest. Ideally, the tracer should be easily detected and quantified, and not affect the system. Usually, kinetic studies are performed in a steady-state, where the rates of input and output for a given unlabeled tracee substance are equal and timeinvariant. Thus, the information provided by the tracer reflects the behavior of the tracee (Barrett et al., 2006). At various times, the amount of tracer is quantified to provide a kinetic curve. Then a mathematical model is constructed to extract all of the information contained in the kinetic curve. By fitting a model to the data, it is possible to calculate the parameters of the model that characterize the flux of molecules between kinetically 


\section{Fasting}

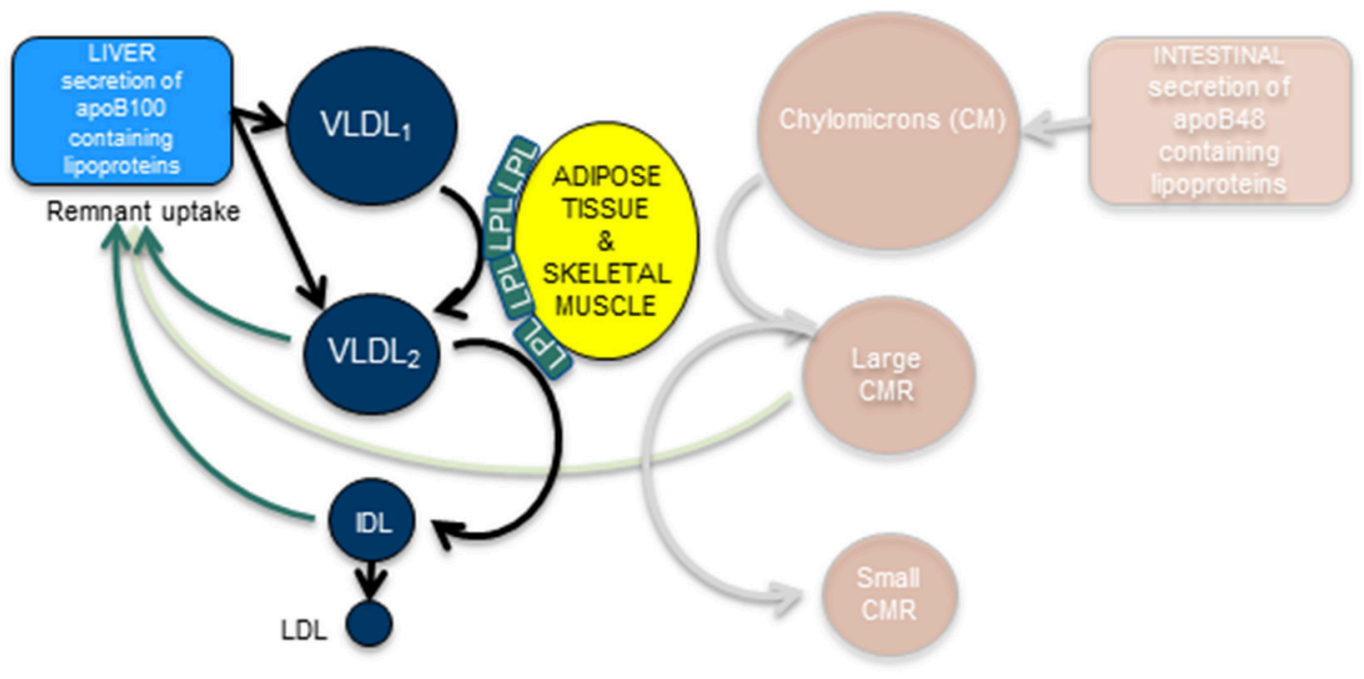

\section{Post-prandial}

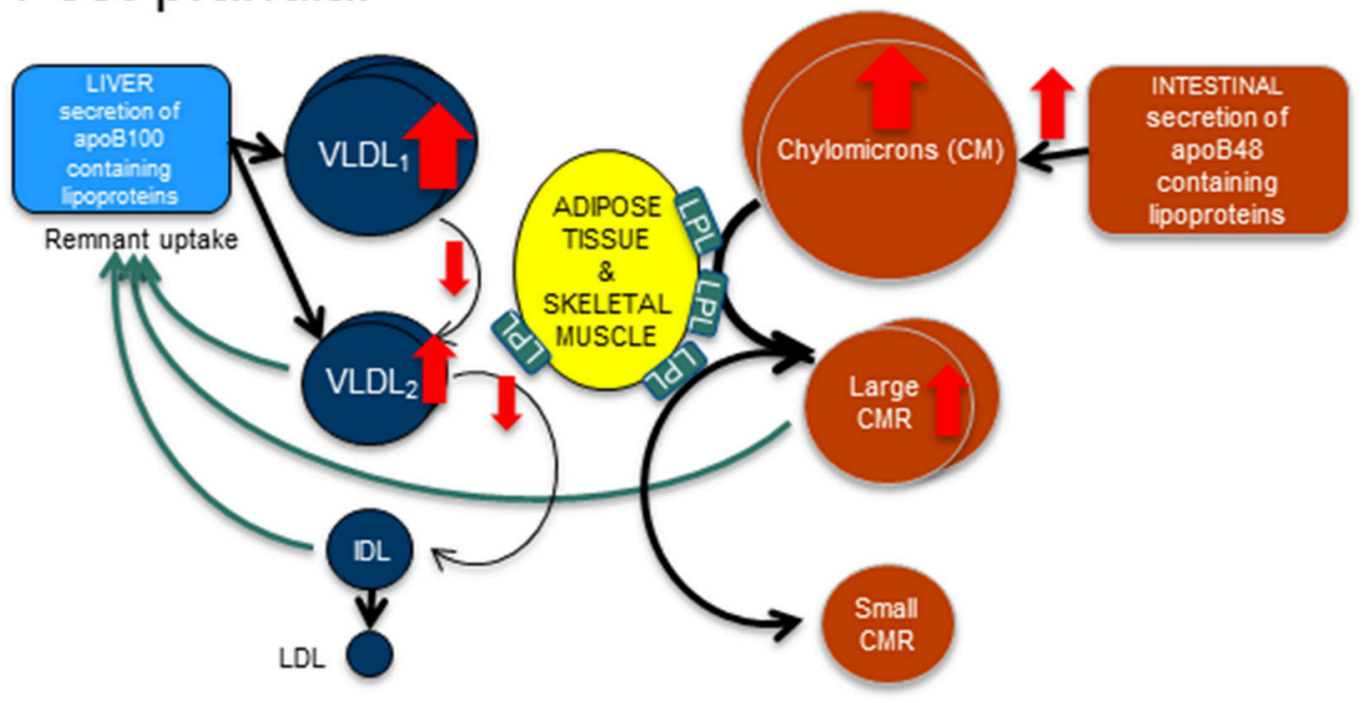

FIGURE 1 | Fasting and post-prandial triglyceride metabolism. In fasting, apoB100 containing lipoproteins are secreted from the liver and are hydrolyzed by lipoprotein lipase (LpL) to form smaller and denser particles. Hence, very low density lipoprotein (VLDL) 1 particles become VLDL2 particles and VLDL2 particles become intermediate density lipoproteins (IDL). After remodeling with cholesterol ester transfer protein and hepatic lipase the end product are the low density lipoproteins (LDL). In the post-prandial phase, the intestine secretes triglyceride rich apoB48 containing lipoproteins, the chylomicrons. After hydrolyzation by LpL, large and small chylomicron remnants (CMR) are formed. This process likely occupies the majority of available LpL, thus resulting in slower turn-over of the apoB100 containing lipoproteins and causing an accumulation of these lipoproteins.

homogeneous pools of molecules. Historically, the radioactive isotopes were used as tracers, but today naturally occurring non-radioactive stable isotopes are almost exclusively used in human studies. The technical advances in mass-spectrometry technologies now permit accurate measurement of stable isotopes in smaller samples and in low concentrations (Barrett et al., 2006; Adiels et al., 2010).
A tracer can be introduced into the system either as a bolus injection or as a constant infusion given immediately after a priming dose. The bolus administration of the tracer is suitable to study kinetics of molecules with a relatively slow rate of turnover since the enrichment curves (the tracer/tracee ratios) after a bolus injection corresponds to the impulse response of the system. Furthermore, it also enables determination of newly synthesized 
particles, since the intracellular precursor enrichment is greater at the start of the study. Primed constant infusions require a longer time to achieve a plateau of isotopic enrichment but can be appropriate for kinetic studies of molecules with rapid turnover.

\section{MODELING OF LIPOPROTEIN METBOLISM}

Mathematical modeling enables us to better understand experimental observations. Predictions derived from model predictions can subsequently be experimentally tested. Toward this goal, known pathways are described by a set of differential equations, thereby allowing quantitative estimates to be derived. In multi-compartment models, molecules of interest move among different compartments of a system. Each compartment is assumed to be a homogeneous entity within which the entities being modeled are equivalent. Multicompartment modeling has proven to yield predictions which are as accurate as those made by physiological models, and the data required can be derived from measurements of tracer/tracee ratios of stable isotopes. Multicompartment models differ in how complex they are. Practically, their design is usually a compromise for what is practically feasible. Too simplified models may not adequately describe the kinetic heterogeneity present within the system. On the other hand, it's hard to generate experimental data for overly complex models.

The power of mathematical modeling to describe the metabolic pathways of lipid and lipoprotein metabolism was first demonstrated by Drs. Berman and Zech (Grundy et al., 1979; Zech et al., 1979). Since then, kinetic studies combined with mathematical modeling have been used to clarify the pathogenesis of impaired lipoprotein metabolism in humans linked to accelerated CVD, obesity, and insulin resistance (Figure 2). The methodology has also been instrumental in testing how efficiently novel drugs improve the dyslipidemia. However, it's important to emphasize that all models are based on several assumptions and simplifications. Therefore, mathematical modeling does not determine the kinetics of lipids directly; rather, they derive an indirect approximation.

\section{MODELS TO STUDY VLDL KINETICS}

Increased levels of apoB-containing lipoproteins are the most important risk-factor for developing CVD. It's therefore clinically relevant to decipher the pathophysiology of impaired metabolism of apoB-containing lipoproteins. Until today most studies have used steady-state models to elucidate VLDL kinetics in the fasting state. However, it can be argued that these studies are not fully physiologically designed since human are in a postprandial state most of the time awake.

To study postprandial lipid metabolism it is necessary to include also intestinal derived lipoproteins. The majority of studies of intestinal lipid metabolism has been conducted in a "constant feeding" regime where small meals are served frequently to achieve a steady state of the intestinal lipoprotein secretion (Lamon-Fava et al., 2007; Sun et al., 2013; Padilla et al., 2014; Tremblay et al., 2014; Xiao et al., 2014). To date, only a few studies have focused on apoB48 metabolism following a

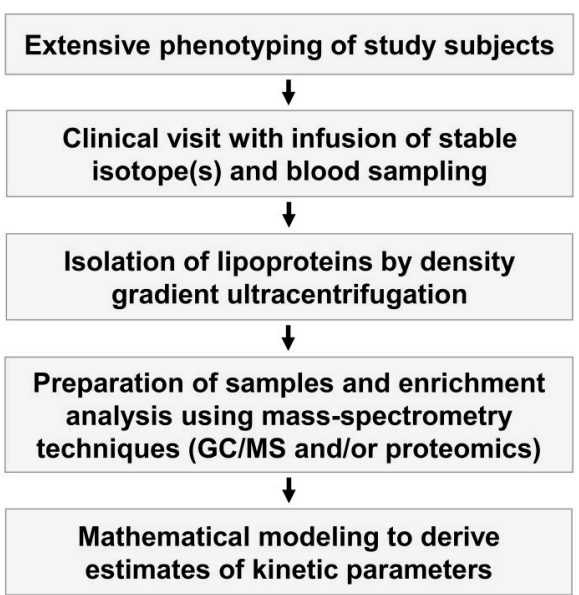

FIGURE 2 | Outline of kinetic tracer studies. In vivo kinetic studies are complex and involve both extensive phenotyping of study subjects, clinical studies where stable isotopes are infused into the study subjects and blood samples are taken, isolation of lipoprotein fractions by ultracentrifugation, enrichment studies of the stable isotopes using different mass spectroscopy techniques, and mathematical modeling of the enrichment curves to derive indices of kinetic variables.

single meal (Wong et al., 2014a,b). No studies have yet linked hepatic and intestinal lipid metabolism in a combined model, and therefore it is not possible to study the interaction of these lipoproteins as depicted in Figure 1. From fat load studies it is obvious that there is a clear interplay between hepatic and intestinal lipoproteins (Adiels et al., 2012). Therefore, such models must include that apoB48- and apoB100-containing particles are cleared from the circulation by common pathways and therefore compete for clearance (Brunzell et al., 1973). During the postprandial phase, other factors need also to be considered such as insulin secretion which may affect hepatic lipoprotein secretion (Lewis et al., 1993; Adiels et al., 2007; Sorensen et al., 2011; Sondergaard et al., 2012).

In steady-state modeling of VLDL kinetics it has been discussed whether VLDL-apoB and VLDL-TG should be modeled in the same integrated model, or if they should be modeled independently. In integrated models the equation for the rate of change of an apoB100 compartment is linked to the rate of change of the corresponding triglyceride compartment size. This procedure of tying together the apoB100 and triglyceride models enhances the precision of the model as a whole. As each particle contains one single copy of apoB100, the model provides an estimate of the lipolytic rates (the loss of triglycerides per time unit), which can then be used as a physiological readout for answering study questions related to dyslipidemia. Drs. Ramakrishnan and Ginsberg recently reported that the VLDL-apoB and VLDL-TG pools in the delipidation cascade have identical rate constants despite different fates and mass distribution (Ramakrishnan and Ginsberg, 2015). These results strongly support integrated steady-state models of VLDL-apoB and VLDL-TG kinetics.

The major predictor of plasma triglycerides are $\mathrm{VLDL}_{1}-\mathrm{TG}$. We therefore developed a multicompartment model that allows 


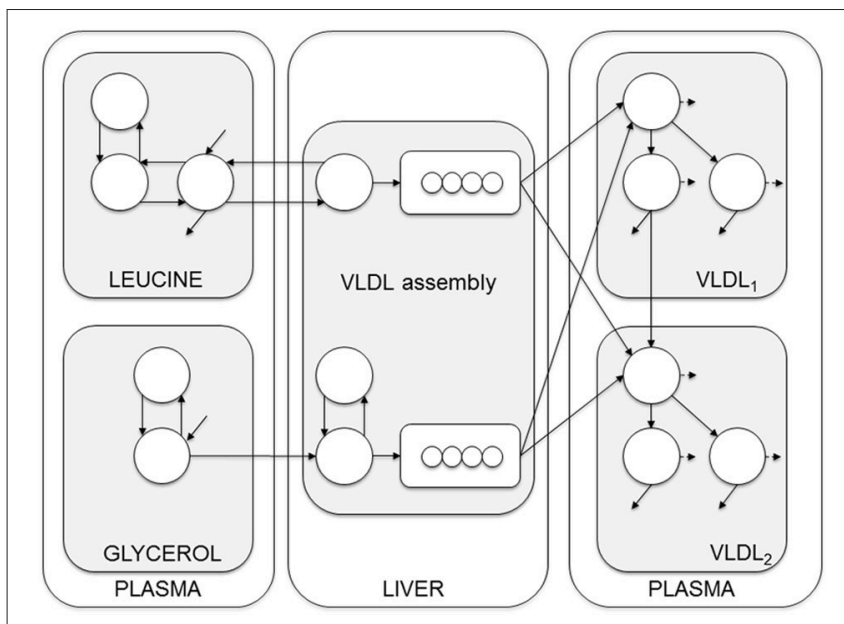

FIGURE 3 | Compartment model of VLDL apoB and triglyceride kinetics. The model includes separate modules for leucine and glycerol. Plasma leucine kinetics is modeled using a four-compartment system that drives the synthesis and secretion of apoB into $\mathrm{VLDL}_{1}$ and $V L D L_{2}$. Plasma glycerol kinetics is modeled using a two-compartment system connected to fast and slow pathways for triglyceride (TG) synthesis. Plasma apoB and triglyceride kinetics are modeled using a four-compartment hydrolysis chain, in which the kinetics of apoB and triglyceride coupled. For each apoB compartment, there is an equivalent compartment for triglyceride. Triglycerides hydrolyzed from VLDL particles are represented by the dashed arrows, and particles lost from the plasma space are represented by the solid arrows. See Adiels et al. (2005) for additional model details.

the kinetics of triglycerides and apoB100 in $\mathrm{VLDL}_{1}$ and $\mathrm{VLDL}_{2}$ to be simultaneously assessed after a bolus injection of glycerol and leucine stable isotopes (Figure 3) (Adiels et al., 2005). Analysis of tracer/trace curves of the stable isotopes in $\mathrm{VLDL}_{1}$ and $\mathrm{VLDL}_{2}$ was used to derive estimates of kinetic parameters using mathematical modeling. By integrating apoB and triglycerides in the model, the triglyceride: apoB ratio of newly produced $\mathrm{VLDL}_{1}$ and $\mathrm{VLDL}_{2}$ particles can be estimated to follow the transfer and removal of lipids (Adiels et al., 2005).

The model can be envisioned as a two-layer model, connected at certain points and is based on the apoB model as described by Packard et al. (1995). The model consists of four parts; plasma leucine, plasma glycerol, the assembly of lipoprotein, and lipoprotein plasma kinetics. The plasma kinetics is modeled by a four-compartment hydrolysis chain, where the apoB and triglyceride kinetics are coupled at the transfer between compartments. Removal from triglyceride compartments consists of both removal of whole particles and removal of triglycerides.

\section{INDIVIDUAL AND POPULATION KINETICS}

Traditionally, parameters for each individual are estimated individually and conclusions are made based on some statistical model applied to the model output. The model complexity ranges from one single compartment models describing VLDL-TG-kinetics (Patterson et al., 2002), to 12 compartments describing the combined apoB and TG kinetics in VLDL1 and
VLDL2 (Adiels et al., 2005). The majority of published studies have used the SAAMII software (The Epsilon Group, US) (Barrett et al., 1998).

As models are becoming more complex and includes more unknown parameters, more data is needed to support the estimation of the model parameters. Individual data sets are also sensitive to loss of data and data quality, which is directly reflected in the variability in the estimated parameters.

Modern modeling techniques combine the mechanistic model (describing the system) with a statistical model (describing the populations). This is an extension to non-linear mixed effects models (NLME) where the non-linear model is the set of ordinary differential equations describing the system. Model parameters are described as random variables drawn from a distribution centered round the population mean. Using data from all individuals, population means and variances as well as individual estimates are calculated for all parameters (Beal and Sheiner, 1982).

The major gain using NLME approaches are that generally the estimated variances are smaller compared to the traditional approach, thus statistical power is greatly increased. Furthermore, such methods have shown produce better results also when data are sparse (Denti et al., 2009; Largajolli et al., 2012). Using these techniques we have recently shown that estimation of lipoprotein kinetics parameters can greatly be improved by an NLME approach (Berglund et al., 2012) (leucine subsystem) and (Berglund et al., 2015) (full system).

To estimate the day-to-day variability in VLDL kinetics and other measures, Magkos et al. repeated a kinetic study in 8 obese men on two occasions 2 months apart (Magkos et al., 2011). Using this data they calculated the sample size needed to detect differences (15-35\%) between two groups during an intervention. For VLDL-TG secretion, $n=15$ was needed in each group to detect a difference of $25 \%$ using an unpaired study design at a study power of $80 \%$. We compared 15 healthy controls and 15 type 2 diabetic subjects and found that using the traditional approach, $n=9$ was needed to detect the difference in $\mathrm{VLDL}_{1}$ secretion. In contrast, using the NLME approach, a sample size of only $n=5$ was needed to detect the same difference (Berglund et al., 2015).

The limitations for these methods are so far that they are very computational intensive and the methods are not yet implemented in traditional software.

\section{PATHOPHYSIOLOGY OF DYSLIPIPIDEMIA IN OBESE SUBJECTS}

To elucidate the pathophysiology of the dyslipidemia in subjects with abdominal obesity, we recently performed lipoprotein kinetic studies in 46 middle-aged well-phenotyped men and women with abdominal obesity and additional cardiometabolic risk factors to clarify determinants of plasma triglyceride concentration (Borén et al., 2015). The results are summarized in Figure 4. The concentration of triglycerides in plasma is determined by the balance between synthesis and removal of $\mathrm{VLDL}_{1}-\mathrm{TG}$. Thus, dual metabolic defects are required to 


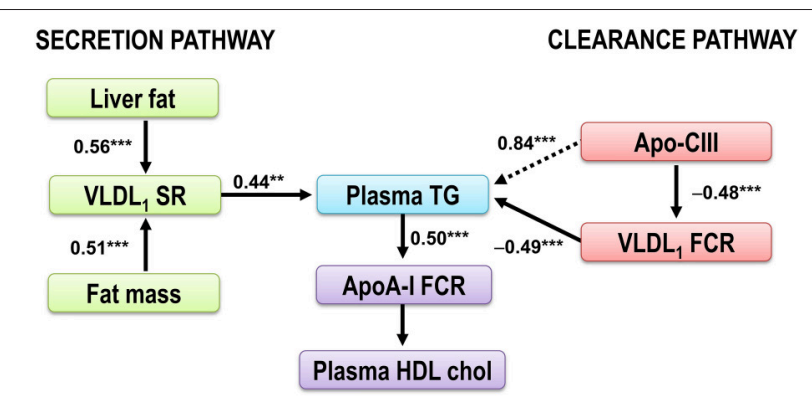

FIGURE 4 | The dysregulation of the VLDL ${ }_{1}-\mathrm{TG}$ and apoAl metabolism in obese subjects. The plasma triglycerides are determined by the balance between synthesis and removal of VLDL 1 -TG, illustrated by a Synthesis pathway and a Clearance pathway. The Synthesis pathway includes liver fat and total fat mass, the two independent predictors of $\mathrm{VLDL}_{1}$-triglyceride secretion rate. In the Clearance pathway, plasma concentration of the LPL inhibitor apoC-III was tightly linked with plasma TG and catabolism of $V_{L D L}$-TG. In addition, apo-CIII was closely related to plasma TG concentration (dotted arrow). This indirect effect of apoC-III on plasma triglycerides is likely explained by effect(s) of apoC-III beyond LPL-independent pathways of triglyceride metabolism (Huard et al., 2005; Sacks et al., 2011). Increased plasma triglycerides are associated with increased HDL catabolism (Pont et al., 2002; Ooi et al., 2005; Chan et al., 2009). Thus, increased plasma triglycerides are closely associated with decreased HDL cholest erol (Verges et al., 2014). Figure modified from Borén et al. (2015). ${ }^{* *} p<0.01 ;{ }^{* * *} p<0.001$.

produce hypertriglyceridemia in obese subjects (Taskinen et al., 2011). To illustrate this, two pathways are shown; a Synthesis pathway and a Clearance pathway. The Synthesis pathway include liver fat and total fat mass since these remained independent predictors of $\mathrm{VLDL}_{1}$-triglyceride secretion rate in a stepwise multivariable regression analysis. In the Clearance pathway, plasma concentration of the LPL inhibitor apoCIII was tightly linked with plasma TG and catabolism of $\mathrm{VLDL}_{1}$-TG. In addition, apo-CIII was closely related to plasma TG concentration. This indirect effect of apoC-III on plasma triglycerides is likely explained by effect(s) of apoC-III beyond LPL-independent pathways of triglyceride metabolism (Huard et al., 2005; Sacks et al., 2011).

HDL cholesterol is closely associated with diabetic dyslipidemia and abdominal obesity (Verges et al., 2014), and low HDL cholesterol is strongly associated with increased cardiovascular risk (Ninomiya et al., 2004). Pharma industry has therefore developed drugs that increase HDL cholesterol. However, clinical studies with these agents have not been successful, and the causative role of HDL is therefore questioned. This indicates that low HDL cholesterol is more a marker of an atherogenic lipoprotein profile. In vivo kinetic studies performed in abdominally obese individuals have shown that low plasma concentration of HDL cholesterol is the consequence of increased HDL catabolism (Pont et al., 2002; Ooi et al., 2005; Chan et al., 2009), and increased plasma triglycerides is closely associated with decreased HDL cholesterol (Verges et al., 2014).

Interestingly, when comparing the Synthesis pathway and the Clearance pathway, indices of catabolism were stronger predictors of plasma triglycerides than parameters of secretion. In a multivariable regression model, $\mathrm{VLDL}_{1}$-TG kinetics explained $76 \%$ of the variation in the total plasma triglycerides. Kinetic parameters of $\mathrm{VLDL}_{1}-\mathrm{TG}$ secretion explained $19-20 \%$ only of the variation in plasma triglyceride concentrations in the study subjects. Thus, in subjects with abdominal obesity and dyslipidemia, the $\mathrm{VLDL}_{1}$-triglyceride clearance is a stronger determinant of the plasma triglyceride concentration than increased secretion of $\mathrm{VLDL}_{1}$ particles. This finding may support combination therapies in subjects with abdominal obesity and dyslipidemia affecting both secretion and catabolism of $\mathrm{VLDL}_{1^{-}}$TG. The results further support apoC-III as a key target for reducing residual cardiovascular risk.

\section{LIVER FAT ACCUMULATION AND CVD}

The observed associations between liver fat plasma triglycerides are in line with earlier studies showing that liver fat content is closely related to triglyceride secretion in different settings and populations (Chan et al., 2010; Taskinen et al., 2011) and a better predictor of triglyceride secretion than intra-abdominal fat (Fabbrini et al., 2009; Magkos et al., 2010). It is also clinically important because of the worrisome increase of non-alcoholic fatty liver disease (NAFLD), defined as hepatic fat accumulation that exceeds $5 \%$ of liver weight in individuals who do not consume significant amounts of alcohol (Neuschwander-Tetri and Caldwell, 2003; Vernon et al., 2011). Approximately 25\% of adults have NAFLD, and its prevalence increases to 70$90 \%$ among adults with obesity or type 2 diabetes (Ray, 2013). Even though NAFLD may progress to severe liver diseases, the most common cause of death in patients with NAFLD is CVD. Several epidemiological studies indicate that NAFLD is not merely a marker of CVD, but may also be actively involved in its pathogenesis (Targher et al., 2008). Thus, the finding that NAFLD is closely linked to overproduction to of $\mathrm{VLDL}_{1}$-TG that drives an atherogenic dyslipidemia characterized with hypertriglyceridemia, HDL-cholesterol, and postprandial hyperlipidemia provide an important explanation for this and indicates that novel treatments reducing liver fat accumulation might be important in preventing CVD (Boren et al., 2013).

\section{CONCLUSION}

Lipid homeostasis is essential for human health but elevated lipid levels are a risk factor for atherosclerosis and thus can lead to symptomatic CVD. Increased lipid levels can be caused by either increased secretion of atherogenic lipoproteins, and/or impaired clearance of lipoproteins from the circulation. The use of in vivo kinetic studies using stable isotopes and mass spectrometry in combination with the development of mathematical models has been critical in advancing understanding of normal and dysregulated lipid metabolism. However, kinetic studies are timeconsuming, expensive and require a high level of expertise. Thus, they are limited to rather few research groups. Future, development will hopefully enable us to optimize the protocols and increase the statistical power, in particular when data are sparse (Denti et al., 2009; Largajolli et al., 2012).

Also, combining kinetic studies with advanced modeling, as genome-scale metabolic modeling (Mardinoglu and Nielsen, 2015; O’Brien et al., 2015), might provide even deeper 
understanding of the metabolic changes and clarify the underlying metabolic perturbations in the occurrence of metabolism related disorders. Genome-scale metabolic models (GEMs) are the collection of the biochemical reactions and associated protein-coding genes, and provide a scaffold for integration of the fluxomics as well as other omics data e.g., proteomics, transcriptomics, metabolomics, and lipidomics. Recently, a functional GEM for the hepatocytes in liver tissue has been reconstructed (Mardinoglu et al., 2014) and its use together with the kinetic studies may provide detailed knowledge for

\section{REFERENCES}

Adiels, M., Larsson, T., Sutton, P., Taskinen, M. R., Boren, J., and Fielding, B. A. (2010). Optimization of N-methyl-N-[tertbutyldimethylsilyl]trifluoroacetamide as a derivatization agent for determining isotopic enrichment of glycerol in very-low density lipoproteins. Rapid Commun. Mass Spectrom. 24, 586-592. doi: 10.1002/rcm.4422

Adiels, M., Matikainen, N., Westerbacka, J., Soderlund, S., Larsson, T., Olofsson, S. O., et al. (2012). Postprandial accumulation of chylomicrons and chylomicron remnants is determined by the clearance capacity. Atherosclerosis 222, 222-228. doi: 10.1016/j.atherosclerosis.2012.02.001

Adiels, M., Olofsson, S. O., Taskinen, M. R., and Boren, J. (2006a). Diabetic dyslipidaemia. Curr. Opin. Lipidol. 17, 238-246. doi: 10.1097/01.mol.0000226115.97436.c0

Adiels, M., Olofsson, S. O., Taskinen, M. R., and Boren, J. (2008). Overproduction of very low-density lipoproteins is the hallmark of the dyslipidemia in the metabolic syndrome. Arterioscler. Thromb. Vasc. Biol. 28, 1225-1236. doi: 10.1161/ATVBAHA.107.160192

Adiels, M., Packard, C., Caslake, M. J., Stewart, P., Soro, A., Westerbacka, J., et al. (2005). A new combined multicompartmental model for apolipoprotein B-100 and triglyceride metabolism in VLDL subfractions. J. Lipid Res. 46, 58-67. doi: 10.1194/jlr.M400108-JLR200

Adiels, M., Taskinen, M. R., Packard, C., Caslake, M. J., Soro-Paavonen, A., Westerbacka, J., et al. (2006b). Overproduction of large VLDL particles is driven by increased liver fat content in man. Diabetologia 49, 755-765. doi: $10.1007 / \mathrm{s} 00125-005-0125-\mathrm{z}$

Adiels, M., Westerbacka, J., Soro-Paavonen, A., Häkkinen, A. M., Vehkavaara, S., Caslake, M. J., et al. (2007). Acute suppression of VLDL1 secretion rate by insulin is associated with hepatic fat content and insulin resistance. Diabetologia 50, 2356-2365. doi: 10.1007/s00125-007-0790-1

Barrett, P. H., Bell, B. M., Cobelli, C., Golde, H., Schumitzky, A., Vicini, P., et al. (1998). SAAM II: simulation, analysis, and modeling software for tracer and pharmacokinetic studies. Metabolism 47, 484-492. doi: 10.1016/S00260495(98)90064-6

Barrett, P. H., Chan, D. C., and Watts, G. F. (2006). Thematic review series: patientoriented research. Design and analysis of lipoprotein tracer kinetics studies in humans. J. Lipid Res. 47, 1607-1619. doi: 10.1194/jlr.R600017-JLR200

Beal, S. L., and Sheiner, L. B. (1982). Estimating population kinetics. Crit. Rev. Biomed. Eng. 8, 195-222.

Berglund, M., Adiels, M., Taskinen, M. R., Boren, J., and Wennberg, B. (2015). Improved Estimation of Human Lipoprotein Kinetics with Mixed Effects Models. PLoS ONE 10:e0138538. doi: 10.1371/journal.pone.0138538

Berglund, M., Sunnåker, M., Adiels, M., Jirstrand, M., and Wennberg, B. (2012). Investigations of a compartmental model for leucine kinetics using non-linear mixed effects models with ordinary and stochastic differential equations. Math. Med. Biol. 29, 361-384. doi: 10.1093/imammb/dqr021

Boren, J., Matikainen, N., Adiels, M., and Taskinen, M. R. (2014). Postprandial hypertriglyceridemia as a coronary risk factor. Clin. Chim. Acta 431, 131-142. doi: 10.1016/j.cca.2014.01.015

Boren, J., Taskinen, M. R., Olofsson, S. O., and Levin, M. (2013). Ectopic lipid storage and insulin resistance: a harmful relationship. J. Intern. Med. 274, 25-40. doi: 10.1111/joim.12071

Borén, J., Watts, G. F., Adiels, M., Söderlund, S., Chan, D. C., Hakkarainen, A., et al. (2015). Kinetic and related determinants of plasma triglyceride concentration understanding the relationship between the genotype-phenotype in different clinical conditions.

\section{ACKNOWLEDGMENTS}

Work by the authors are supported by grants from the Swedish Medical Research Council, the Swedish Foundation for Strategic Research, the Sigrid Juselius Foundation and EUproject RESOLVE (FP7-HEALTH-INNOVATION-2012-1, Nr. 305707).

in abdominal obesity. Multicenter tracer kinetic study. Arterioscler. Thromb. Vasc. Biol. 35, 2218-2224. doi: 10.1161/ATVBAHA.115.305614

Brunzell, J. D., Hazzard, W. R., Porte, D. Jr., and Bierman, E. L. (1973). Evidence for a common, saturable, triglyceride removal mechanism for chylomicrons and very low density lipoproteins in man. J. Clin. Invest. 52, 1578-1585. doi: 10.1172/JCI107334

Chan, D. C., Barrett, P. H., Ooi, E. M., Ji, J., Chan, D. T., and Watts, G. F. (2009). Very low density lipoprotein metabolism and plasma adiponectin as predictors of high-density lipoprotein apolipoprotein A-I kinetics in obese and nonobese men. J. Clin. Endocrinol. Metab. 94, 989-997. doi: 10.1210/jc.2008-1457

Chan, D. C., Barrett, P. H., and Watts, G. F. (2004a). Lipoprotein transport in the metabolic syndrome (Part I): methodological aspects of stable isotope kinetic studies. Clin. Sci. (Lond.) 107, 221-232. doi: 10.1042/CS20040108

Chan, D. C., Barrett, P. H., and Watts, G. F. (2004b). Lipoprotein transport in the metabolic syndrome (Part II): Pathophysiological and interventional studies employing stable isotopy and modelling methods. Clin. Sci. (Lond.) 107, 233-249. doi: 10.1042/CS20040109

Chan, D. C., Watts, G. F., Gan, S., Wong, A. T., Ooi, E. M., and Barrett, P. H. (2010). Nonalcoholic fatty liver disease as the transducer of hepatic oversecretion of very-low-density lipoprotein-apolipoprotein B-100 in obesity. Arterioscler. Thromb. Vasc. Biol. 30, 1043-1050. doi: 10.1161/ATVBAHA.109.202275

Denti, P., Bertoldo, A., Vicini, P., and Cobelli, C. (2009). Nonlinear mixed effects to improve glucose minimal model parameter estimation: a simulation study in intensive and sparse sampling. IEEE Trans. Biomed. Eng. 56, 2156-2166. doi: 10.1109/TBME.2009.2020171

Fabbrini, E., Magkos, F., Mohammed, B. S., Pietka, T., Abumrad, N. A., Patterson, B. W., et al. (2009). Intrahepatic fat, not visceral fat, is linked with metabolic complications of obesity. Proc. Natl. Acad. Sci. U.S.A. 106, 15430-15435. doi: 10.1073/pnas.0904944106

Grundy, S. M., Mok, H. Y. I., Zech, L., Steinberg, D., and Berman, M. (1979). Transport of very low density lipoprotein triglycerides in varying degrees of obesity and hypertriglyceridemia. J. Clin. Invest. 63, 1274-1283. doi: 10.1172/JCI109422

Hiukka, A., Fruchart-Najib, J., Leinonen, E., Hilden, H., Fruchart, J. C., and Taskinen, M. R. (2005). Alterations of lipids and apolipoprotein CIII in very low density lipoprotein subspecies in type 2 diabetes. Diabetologia 48, 1207-1215. doi: 10.1007/s00125-005-1753-z

Huard, K., Bourgeois, P., Rhainds, D., Falstrault, L., Cohn, J. S., and Brissette, L. (2005). Apolipoproteins C-II and C-III inhibit selective uptake of low- and high-density lipoprotein cholesteryl esters in HepG2 cells. Int. J. Biochem. Cell Biol. 37, 1308-1318. doi: 10.1016/j.biocel.2005.01.005

Hussain, M. M. (2014). Intestinal lipid absorption and lipoprotein formation. Curr. Opin. Lipidol. 25, 200-206. doi: 10.1097/MOL.0000000000000084

Lamon-Fava, S., Diffenderfer, M. R., Barrett, P. H., Buchsbaum, A., Matthan, N. R., Lichtenstein, A. H., et al. (2007). Effects of different doses of atorvastatin on human apolipoprotein B-100, B-48, and A-I metabolism. J. Lipid Res. 48, 1746-1753. doi: 10.1194/jlr.M700067-JLR200

Largajolli, A., Bertoldo, A., and Cobelli, C. (2012). Identification of the glucose minimal model by stochastic nonlinear-mixed effects methods. Conf. Proc. IEEE Eng. Med. Biol. Soc. 2012, 5482-5485. doi: 10.1109/embc.2012.6347235

Lewis, G. F., Uffelman, K. D., Szeto, L. W., and Steiner, G. (1993). Effects of acute hyperinsulinemia on VLDL triglyceride and VLDL apoB production in normal weight and obese individuals. Diabetes 42, 833-842. doi: 10.2337/diab. 42.6 .833 
MacArthur, J. M., Bishop, J. R., Stanford, K. I., Wang, L., Bensadoun, A., Witztum, J. L., et al. (2007). Liver heparan sulfate proteoglycans mediate clearance of triglyceride-rich lipoproteins independently of LDL receptor family members. J. Clin. Invest. 117, 153-164. doi: 10.1172/JCI29154

Magkos, F., Fabbrini, E., Korenblat, K., Okunade, A. L., Patterson, B. W., and Klein, S. (2011). Reproducibility of glucose, fatty acid and VLDL kinetics and multiorgan insulin sensitivity in obese subjects with non-alcoholic fatty liver disease. Int. J. Obes. (Lond). 35, 1233-1240. doi: 10.1038/ijo.2010.265

Magkos, F., Fabbrini, E., Mohammed, B. S., Patterson, B. W., and Klein, S. (2010). Increased whole-body adiposity without a concomitant increase in liver fat is not associated with augmented metabolic dysfunction. Obesity (Silver Spring) 18, 1510-1515. doi: 10.1038/oby. 2010.90

Mahley, R. W., Innerarity, T. L., Rall, S. C. Jr., and Weisgraber, K. H. (1984). Plasma lipoproteins: apolipoprotein structure and function. J. Lipid Res. 25, 1277-1294.

Mahley, R. W., and Ji, Z. S. (1999). Remnant lipoprotein metabolism: key pathways involving cell-surface heparan sulfate proteoglycans and apolipoprotein E. J. Lipid Res. 40, 1-16.

Mardinoglu, A., Agren, R., Kampf, C., Asplund, A., Uhlen, M., and Nielsen, J. (2014). Genome-scale metabolic modelling of hepatocytes reveals serine deficiency in patients with non-alcoholic fatty liver disease. Nat. Commun. 5, 3083. doi: $10.1038 /$ ncomms 4083

Mardinoglu, A., and Nielsen, J. (2015). New paradigms for metabolic modeling of human cells. Curr. Opin. Biotechnol. 34, 91-97. doi: 10.1016/j.copbio.2014.12.013

Neuschwander-Tetri, B. A., and Caldwell, S. H. (2003). Nonalcoholic steatohepatitis: summary of an AASLD single topic conference. Hepatology 37, 1202-1219. doi: 10.1053/jhep.2003.50193

Ninomiya, J. K., L'Italien, G., Criqui, M. H., Whyte, J. L., Gamst, A., and Chen, R. S. (2004). Association of the metabolic syndrome with history of myocardial infarction and stroke in the Third National Health and Nutrition Examination Survey. Circulation 109, 42-46. doi: 10.1161/01.CIR.0000108926.04022.0C

O’Brien, E. J., Monk, J. M., and Palsson, B. O. (2015). Using genomescale models to predict biological capabilities. Cell 161, 971-987. doi: 10.1016/j.cell.2015.05.019

Ooi, E. M., Watts, G. F., Farvid, M. S., Chan, D. C., Allen, M. C., Zilko, S. R., et al. (2005). High-density lipoprotein apolipoprotein A-I kinetics in obesity. Obes. Res. 13, 1008-1016. doi: 10.1038/oby.2005.118

Packard, C. J., Gaw, A., Demant, T., and Shepherd, J. (1995). Development and application of a multicompartmental model to study very low density lipoprotein subfraction metabolism. J. Lipid Res. 36, 172-187.

Padilla, N., Maraninchi, M., Béliard, S., Berthet, B., Nogueira, J. P., Wolff, E., et al. (2014). Effects of bariatric surgery on hepatic and intestinal lipoprotein particle metabolism in obese, nondiabetic humans. Arterioscler. Thromb. Vasc. Biol. 34, 2330-2337. doi: 10.1161/ATVBAHA.114.303849

Patterson, B. W., Mittendorfer, B., Elias, N., Satyanarayana, R., and Klein, S. (2002). Use of stable isotopically labeled tracers to measure very low density lipoprotein-triglyceride turnover. J. Lipid Res. 43, 223-233.

Pont, F., Duvillard, L., Florentin, E., Gambert, P., and Verges, B. (2002). Highdensity lipoprotein apolipoprotein A-I kinetics in obese insulin resistant patients. An in vivo stable isotope study. Int. J. Obes. Relat. Metab. Disord. 26, 1151-1158. doi: 10.1038/sj.ijo.0802070

Powell, L. M., Wallis, S. C., Pease, R. J., Edwards, Y. H., Knott, T. J., and Scott, J. (1987). A novel form of tissue-specific RNA processing produces apolipoprotein-B48 in intestine. Cell 50, 831-840. doi: 10.1016/00928674(87)90510-1

Ramakrishnan, S., and Ginsberg, H. (2015). "Modeling the VLDL delipidation cascade: apolipoprotein B and triglyceride pools have identical rate constants despite different fates and mass distributions," in 17th ISA Congress (Amsterdam)

Ray, K. (2013). NAFLD-the next global epidemic. Nat. Rev. Gastroenterol. Hepatol. 10, 621. doi: 10.1038/nrgastro.2013.197

Sacks, F. M., Zheng, C., and Cohn, J. S. (2011). Complexities of plasma apolipoprotein C-III metabolism. J. Lipid Res. 52, 1067-1070. doi: 10.1194/jlr.E015701

Segrest, J. P., Jones, M. K., De Loof, H., and Dashti, N. (2001). Structure of apolipoprotein B-100 in low density lipoproteins. J. Lipid Res. 42, 1346-1367.

Sondergaard, E., Sorensen, L. P., Rahbek, I., Gormsen, L. C., Christiansen, J. S., and Nielsen, S. (2012). Postprandial VLDL-triacylglycerol secretion is not suppressed in obese type 2 diabetic men. Diabetologia 55, 2733-2740. doi: $10.1007 / \mathrm{s} 00125-012-2624-\mathrm{z}$

Sørensen, L. P., Andersen, I. R., Søndergaard, E., Gormsen, L. C., Schmitz, O., Christiansen, J. S., et al. (2011). Basal and insulin mediated VLDL-triglyceride kinetics in type 2 diabetic men. Diabetes 60, 88-96. doi: 10.2337/db10-0564

Sun, F., Stolinski, M., Shojaee-Moradie, F., Lou, S., Ma, Y., Hovorka, R., et al. (2013). A novel method for measuring intestinal and hepatic triacylglycerol kinetics. Am. J. Physiol. Endocrinol. Metab. 305, E1041-E1047. doi: 10.1152/ajpendo.00105.2013

Targher, G., Marra, F., and Marchesini, G. (2008). Increased risk of cardiovascular disease in non-alcoholic fatty liver disease: causal effect or epiphenomenon? Diabetologia 51, 1947-1953. doi: 10.1007/s00125-008-1135-4

Taskinen, M. R. (2003). Diabetic dyslipidaemia: from basic research to clinical practice. Diabetologia 46, 733-749. doi: 10.1007/s00125-003-1111-y

Taskinen, M. R. (2005). Type 2 diabetes as a lipid disorder. Curr. Mol. Med. 5, 297-308. doi: 10.2174/1566524053766086

Taskinen, M. R., Adiels, M., Westerbacka, J., Soderlund, S., Kahri, J., Lundbom, N., et al. (2011). Dual metabolic defects are required to produce hypertriglyceridemia in obese subjects. Arterioscler. Thromb. Vasc. Biol. 31, 2144-2150. doi: 10.1161/ATVBAHA.111.224808

Taskinen, M. R., and Boren, J. (2015). New insights into the pathophysiology of dyslipidemia in type 2 diabetes. Atherosclerosis 239, 483-495. doi: 10.1016/j.atherosclerosis.2015.01.039

Tremblay, A. J., Lamarche, B., Labonte, M. E., Lepine, M. C., Lemelin, V., and Couture, P. (2014). Dietary medium-chain triglyceride supplementation has no effect on apolipoprotein B-48 and apolipoprotein B-100 kinetics in insulin-resistant men. Am. J. Clin. Nutr. 99, 54-61. doi: 10.3945/ajcn.113. 068767

Verges, B., Adiels, M., Boren, J., Barrett, P. H., Watts, G. F., Chan, D., et al. (2014). Interrelationships between the kinetics of VLDL subspecies and HDL catabolism in abdominal obesity: a multicenter tracer kinetic study. J. Clin. Endocrinol. Metab. 99, 4281-4290. doi: 10.1210/jc.2014-2365

Vernon, G., Baranova, A., and Younossi, Z. M. (2011). Systematic review: the epidemiology and natural history of non-alcoholic fatty liver disease and nonalcoholic steatohepatitis in adults. Aliment. Pharmacol. Ther. 34, 274-285. doi: 10.1111/j.1365-2036.2011.04724.x

Williams, K. J. (2008). Molecular processes that handle-and mishandle-dietary lipids. J. Clin. Invest. 118, 3247-3259. doi: 10.1172/JCI35206

Williams, K. J., and Chen, K. (2010). Recent insights into factors affecting remnant lipoprotein uptake. Curr. Opin. Lipidol. 21, 218-228. doi: 10.1097/MOL.0b013e328338cabc

Wong, A. T., Chan, D. C., Barrett, P. H., Adams, L. A., and Watts, G. F. (2014a). Effect of omega-3 fatty acid ethyl esters on apolipoprotein B-48 kinetics in obese subjects on a weight-loss diet: a new tracer kinetic study in the postprandial state. J. Clin. Endocrinol. Metab. 99, E1427-E1435. doi: 10.1210/jc. 2013-4037

Wong, A. T., Chan, D. C., Pang, J., Watts, G. F., and Barrett, P. H. (2014b). Plasma apolipoprotein B-48 transport in obese men: a new tracer kinetic study in the postprandial state. J. Clin. Endocrinol. Metab. 99, E122-E126. doi: $10.1210 /$ jc.2013-2477

Xiao, C., Dash, S., Morgantini, C., Patterson, B. W., and Lewis, G. F. (2014). Sitagliptin, a DPP-4 inhibitor, acutely inhibits intestinal lipoprotein particle secretion in healthy humans. Diabetes 63, 2394-2401. doi: 10.2337/db13-1654

Zech, L. A., Grundy, S. M., Steinberg, D., and Berman, M. (1979). Kinetic model for production and metabolism of very low density lipoprotein triglycerides. Evidence for a slow production pathway and results for normolipidemic subjects. J. Clin. Invest. 63, 1262-1273. doi: 10.1172/JCI109421

Conflict of Interest Statement: The authors declare that the research was conducted in the absence of any commercial or financial relationships that could be construed as a potential conflict of interest.

Copyright (c) 2015 Adiels, Mardinoglu, Taskinen and Borén. This is an open-access article distributed under the terms of the Creative Commons Attribution License (CC $B Y)$. The use, distribution or reproduction in other forums is permitted, provided the original author(s) or licensor are credited and that the original publication in this journal is cited, in accordance with accepted academic practice. No use, distribution or reproduction is permitted which does not comply with these terms. 